\title{
Phytophthora parasitica Effector PpRxLR2 Suppresses Nicotiana benthamiana Immunity
}

\author{
R. J. D. Dalio, ${ }^{1}$ H. J. Maximo, ${ }^{1}$ T. S. Oliveira, ${ }^{1}$ R. O. Dias, ${ }^{2}$ M. C. Breton, ${ }^{1}$ H. Felizatti, ${ }^{3}$ and M. Machado ${ }^{1,+}$ \\ ${ }^{1}$ Biotechnology Laboratory, Centro de Citricultura Sylvio Moreira/Instituto Agronômico, Cordeirópolis, SP, Brazil; ${ }^{2}$ Instituto de \\ Química, Universidade de São Paulo USP, São Paulo, SP, Brazil; and ${ }^{3}$ Instituto de Matemática, Física e Computação Científica, \\ Universidade Estadual de Campinas Unicamp, Campinas, SP, Brazil
}

Accepted 19 November 2017.

\begin{abstract}
Phytophthora species secrete several classes of effector proteins during interaction with their hosts. These proteins can have multiple functions including modulation of host physiology and immunity. The RxLR effectors have the ability to enter plant cells using the plant machinery. Some of these effectors have been characterized as immunity suppressors; however, very little is known about their functions in the interaction between Phytophthora parasitica and its hosts. Using a bioinformatics pipeline, we have identified 172 candidate RxLR effectors (CREs) in the isolate IAC 01_95 of P. parasitica. Of these 172 CREs, 93 were found to be also present in eight other genomes of $P$. parasitica, isolated from different hosts and continents. After transcriptomics and gene expression analysis, we have found five CREs to be up-regulated in in-vitro and in-planta samples. Subsequently, we selected three CREs for functional characterization in the model plant Nicotiana benthamiana. We show that PpRxLR2 is able to completely suppress INF-1induced cell death, whereas PpRxLR3 and PpRxLR5 moderately suppressed $N$. benthamiana immunity in a less-extensive manner. Moreover, we confirmed the effector-triggered susceptibility activity of these proteins after transient transformation and infection of $N$. benthamiana plants. All three CREs enhanced virulence of $\boldsymbol{P}$. parasitica during the interaction with $N$. benthamiana. These effectors, in particular PpRxLR2, can be targeted for the development of biotechnology-based control strategies of $P$. parasitica diseases.
\end{abstract}

Plant-pathogenic oomycetes are responsible for economic losses in agriculture and forestry and for damage in natural ecosystems. Notable oomycete species include Phytophthora infestans, the causative agent of late blight in potato, which was responsible for the Irish famine in the 18th century, $P$. sojae, responsible for the root rot and stem rot of soybean, $P$. ramorum, which causes sudden oak death, $P$. capsici, responsible for several diseases in plants from the Curcubitaceae, Fabaceae, and Solanaceae families, and $P$. parasitica, which

\section{R. J. D. Dalio and H. J. Maximo contributed equally to this work. \\ ${ }^{\dagger}$ Corresponding author: M. Machado; E-mail: marcos@ccsm.br}

Funding: The work was supported by Conselho Nacional de Desenvolvimento Científico e Tecnológico grant 445390/2014-0 and 465440/2014-2 and Fundação de Amparo à Pesquisa do Estado de São Paulo grant 2015/14498-6.

*The $\boldsymbol{e}$-Xtra logo stands for "electronic extra" and indicates that nine supplementary files are published online.

○ 2018 The American Phytopathological Society has a remarkably wide host range capable of infecting more than 250 genera from over 93 families of plants (Kamoun et al. 2015).

Phytophthora parasitica Dastur (syn. Phytophthora nicotianae Breda de Haan) is cosmopolitan and has been isolated from multiple plants and habitats, ranging from crop plants, forest trees, medicinal herbs, natural ecosystems, and waterrecycling systems (Panabieres et al. 2016). It is particularly aggressive toward citrus plants, causing root rot and gummosis. It is estimated that Phytophthora diseases are responsible for losses of $\$ 30$ to 60 million per year in citrus orchards in Florida (Graham and Feichtenberger 2015). It also infects plants from the Nicotianae family, including the model plant Nicotiana benthamiana. Currently, no efficient and sustainable method to control $P$. parasitica diseases is available.

It is known that Phytophthora species secrete several classes of effector proteins during interaction with their hosts (Hogenhout et al. 2009; Kamoun 2006). These proteins can have several functions, including the modulation of host physiology and immunity. When effectors suppress plant defenses, this phenomenon is called effector-triggered susceptibility. Plants, in turn, can recognize pathogen effectors through specialized proteins, named $\mathrm{R}$ proteins, and activate their defense system. This is known as effector-triggered immunity (ETI). Another layer of defense, also based on recognition, called pathogen-associated molecular pattern (PAMP)-triggered immunity (PTI) also play an important role in the plant defense system. Through pathogen or PAMP-recognition receptors, plants can recognize PAMPs and activate defenses. Both ETI and PTI can lead to a hypersensitive response (HR) (Thomma et al. 2011). HR is a type of programmed cell death (PCD) that happens after an oxidative burst, in which reactive oxygen species (ROS) are locally released upon pathogen recognition (Künstler et al. 2016). This oxidative stress may kill plant tissues but, also, pathogen cells that may contain the infection.

Millions of years in coevolution have created an arms race between plants and pathogens. On one side, plants must create efficient forms of pathogen recognition, on the other side, pathogens must develop effectors to suppress PTI and ETI (Jones and Dangl 2006). The result of this arms race is a complex biochemical communication system during plantpathogen interactions. Thanks to high-throughput sequencing technologies, several key players in this communication system, such as effectors, have been found. Phytophthora spp. for instance, have been reported to secrete cytoplasmic effectors, namely, RxLR and Crinkler effectors (Whisson et al. 2007). These families of effectors carry special amino-acid motifs required for their entry into cells using the host machinery. A 
few of these cytoplasmic effectors have already been characterized to play a role in pathogenicity, either eliciting or suppressing defenses (Bos et al. 2009; Stam et al. 2013; Tyler et al. 2006; Zhang et al. 2015).

The RxLR effectors are defined as being modular proteins carrying signal peptides and a conserved RxLR-dEER motif at the N-terminal region. This conserved amino-acid motif (arginine, any amino acid, leucine, and arginine) allows scrutinizing these effectors through bioinformatic pipelines within sequenced genomes, uncovering a solid set of candidates (Anderson et al. 2015). Putative RxLR effectors have already been shown for some Phytophthora species, such as $P$. infestans and $P$. sojae (King et al. 2014; Song et al. 2013). Among these candidates, some have been shown to be involved in pathogenicity. For instance, Avr3a of P. infestans was shown to suppress PCD induced by INF-1 elicitin, a protein also secreted by $P$. infestans that has PAMP features and elicits cell death in Nicotianae species (Armstrong et al. 2005; Bos et al. 2010; Gilroy et al. 2011). In $P$. sojae, in silico analysis identified nearly 400 putative RxLR effectors and 169 candidates were functionally tested, resulting in 22 candidates that were validated as able to suppress INF-1-induced cell death (Wang et al. 2011). In addition, the RxLR effector PSE1 (penetration-specific effector-1) of $P$. parasitica was functionally characterized in Nicotianae and Arabidopsis thaliana plants (Evangelisti et al. 2013). The authors found that PSE1 is secreted during penetration and is facilitated by locally modulating auxin metabolism. PSE1 also suppressed PCD induced by the proapoptotic protein BAX (bcl-2-associated $X$ protein). To our knowledge, there is no information in literature exploring the RxLR-candidate set of $P$. parasitica, and, with exception of PSE1, the role of these effectors to pathogenicity remains elusive.

It is fundamental to understand the function of effectors during host-pathogen interactions in order to create sustainable, biotechnology-driven control strategies of diseases. Despite recent advances in the field, very little is known about the role of RxLR effectors in Phythophthora-plant interactions, especially for $P$. parasitica. In this study, we have identified a RxLR candidate effectors repertoire of $P$. parasitica and have expressed three of them, PpRxLR2, PpRxLR3, and PpRxLR5, in $N$. benthamiana leaves through Agrobacterium tumefaciens transient transformation. We have found that PpRxLR2 completely suppressed the PCD induced by the INF-1 elicitin of $P$. infestans. PpRxLR3 and PpRxLR5 also suppressed plant defenses but to a lesser extent when compared with PpRxLR2. However, expression of all three effectors enhanced susceptibility of $N$. benthamiana to $P$. parasitica, indicating that these effectors positively contribute to the pathogen virulence.

\section{RESULTS}

\section{CREs of $P$. parasitica.}

There are currently 11 sequenced genomes of $P$. parasitica isolates obtained from different regions and hosts available on public databases. We took advantage of nine genomes (Table 1) that were sequenced in a joint consortium (therefore, all nine were sequenced under the same conditions) and used these data to search for secreted CREs. We applied a bioinformatics pipeline designed to filter sequences that contains both signal peptides and RxLR-EER motifs (Fig. 1A). The estimated number of genes in these isolates is similar, ranging only $9 \%$ from the highest to the lowest. The estimated number of CREs are also similar, with the isolate P1569.1 from citrus presenting the higher number (217) and the CJ05E6.1, isolated from tobacco, the lowest (167). The object of this study, isolate IAC 01_95, also obtained from citrus, displayed 172 CREs (Table 1). A complete list of all 172 candidates containing its amino-acid sequences is available in the Supplementary Material S1.

Further, we searched for the occurrence of the 172 CREs from the IAC 01_95 isolate in the datasets of the other eight isolates. The parameters used were i) more than $95 \%$ identity and ii) more than $50 \%$ coverage in the nucleotide sequences. We found that another isolate from citrus, P1569.1, shares the highest similarity (151 hits, $87 \%$ similarity), whereas isolate INRA-310 from tobacco shares the lowest similarity (132 hits, $76 \%$ similarity), when compared with IAC 01_95 (Fig. 1B). In total, 93 of 172 CREs were identified in all isolates and are listed in Supplementary Material S2. These sequences can be considered as "core RxLR effectors" of $P$. parasitica. Despite different selection pressures coming from the distinct hosts and ecosystems in which these $P$. parasitica were isolated, the number of shared CRE sequences remained high. Altogether, this result indicates that RxLR effectors should play an important role for pathogen fitness, regardless of one or both their source region or host.

Additionally, a cluster analysis was performed and a dendrogram was built with the sequence pool that was not present in the genome of at least one isolate (Fig. 1C). This analysis shows the genomes clustering according to the presence or absence of homolog sequences to the 172 CREs, highlighting the most similar (P1569.1) and the most distinct isolate (INRA310) to IAC 01_95. In general, tobacco isolates, except CJ01A1, were the most distinct in relation to IAC 01_95, with 10 of the 172 CREs being not identified in any of the isolates (INRA-310, CJ05E6, CJ02B3, and CJ01A1). This observation shows the importance of the host to promote evolutionary selective pressures over RxLR effectors and to create a diverse reservoir of these molecules. Note that three PpRxLRs, namely, 149,30 , and 57, are exclusively found in isolate IAC 01_95. These particular three CREs may play a role in the adaptation of this isolate, either as a virulence factor in citrus or as an adaptation factor to tropical climates. These candidates will be targets for future functional characterization.

Similarly, other plant-pathogenic oomycetes have been screened for the presence of CREs (Table 2). P. infestans, a species with a narrow host range, has the biggest genome size (240 Mb) and the highest number of RxLR effectors (716). $P$. sojae, also with a narrow host range, has a significantly

Table 1. Estimated number of genes and candidate RxLR effectors (CREs) in Phytophthora parasitica genomes

\begin{tabular}{|c|c|c|c|c|}
\hline$P$. parasitica isolates & No. of genes & $\%$ & Host & No. of CREs \\
\hline P1569.1 & 23.159 & 100 & Citrus & 217 \\
\hline P1976.1 & 23.127 & 99.8 & Tomato & 209 \\
\hline INRA-310.3 & 23.121 & 99.8 & Tobacco & 205 \\
\hline P10297.1 & 23.114 & 99.8 & Dieffenbachia exotica & 212 \\
\hline CJ01A1.1 & 23.061 & 99.5 & Tobacco & 213 \\
\hline CJ05E6.1 & 21.812 & 94.1 & Tobacco & 167 \\
\hline IAC_01/95 & 21.789 & 94.0 & Citrus & 172 \\
\hline CHvinca01.1 & 21.679 & 93.6 & Periwinkle & 225 \\
\hline CJ02B3.1 & 21.085 & 91.0 & Tobacco & 168 \\
\hline
\end{tabular}


smaller genome $(95 \mathrm{Mb})$ but, relatively, presents a high number of CREs (672), similar to what was observed in P. ramorum. On the other hand, $P$. parasitica, a pathogen with the broadest host range has a similar genome size of $P$. sojae $(95 \mathrm{Mb})$ but only 172 CREs (identified herein), suggesting that the number of CREs present in a genome might be more related to the source host rather than the genome size.

It is known that, during infection, many effectors target subcellular compartments such as mitochondrion, chloroplasts, and nuclei. To further gain insights into the possible functions of CREs inside plant cells, we predicted the subcellular localization of the 172 CREs from $P$. parasitica with the software LOCALIZER (Sperschneider et al. 2017) (Supplementary Material S3). The software searches for the presence of mitochondrion and chloroplast transit peptides (mTPs and cTPs, respectively) and nuclear localization signals (NLS). In our analysis, it was found that only one CRE has cTP, two CREs have mTPs, and one CRE has both mTP and NLS, while 32 CREs $(18.6 \%)$ are guided to the nucleus.

A neighbor-joining phylogenetic tree was constructed for all 172 CREs selected from $P$. parasitica isolate IAC 01_95 (Fig. 2). The protein sequences were clustered in several distinct clades.
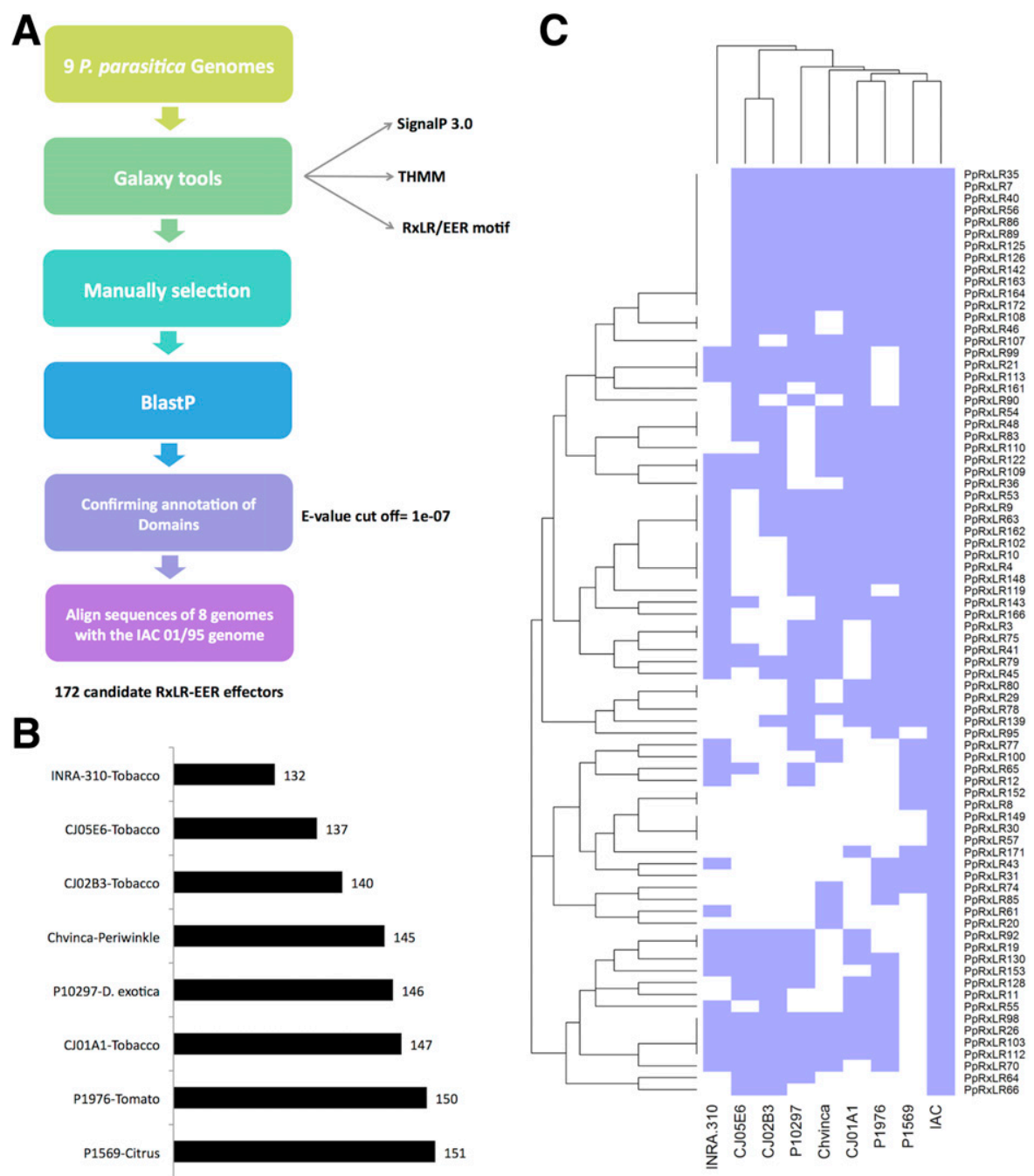

Fig. 1. A, Bioinformatic pipeline designed to predict and compare candidate RxLR effectors (CREs) in the genomes of Phytophthora parasitica. B, Occurrence of 172 CREs from the isolate IAC 01_95 in eight other sequenced genomes of $P$. parasitica (P1569.1, P1976.1, INRA-310.3, P10297.1, CJ01A1.1, CJ05E6.1, CHvinca01.1, CJ02B3.1). Parameters were more than 95\% identity and 50\% coverage. C, A dendrogram showing the homology between sequences of the RxLR candidates in the genomes of $P$. parasitica.

Table 2. Estimated number of genes and candidate RxLR effectors (CREs) in Phytophthora spp. genomes ${ }^{\mathrm{a}}$

\begin{tabular}{lcccc}
\hline Phytophthora spp. & Genome size $(\mathbf{M b})$ & No. of genes & No. of CREs & Reference \\
\hline Hyaloperonospora parasitica & 75 & NA & 149 & Lamour et al. 2007 \\
P. capsici & 65 & 12.011 & 420 & JGI \\
P. infestans & 240 & 22.658 & 716 & Broad Institute \\
P. ramorum & 65 & 15.743 & 531 & JGI \\
P. sojae & 95 & 19.027 & 672 & JGI \\
P. palmivora & 126 & NA & 140 & Ali et al. 2017 \\
P. parasitica & 95 & 21.789 & 172 & \\
\hline
\end{tabular}

${ }^{a} \mathrm{NA}=$ not applicable. JGI = Joint Genome Institute. 
Based on this phylogeny and the subsequent gene-expression analysis, three sequences from distinct clades (PpRxLR2, PpRxLR3, and PpRxLR5) were chosen for functional characterization by $A$. tumefaciens transient expression assays and tridimensional structure predictions.

The genome architecture of isolate IAC 01_95 of P. parasitica is shown in Figure 3. This analysis groups genes regarding their intergenic distances, evidencing dense and sparse regions in the genome. In contrast to $P$. infestans, which is frequently called a two-speed genome (discontinuous distribution of gene density) (Haas et al. 2009), P. parasitica isolate IAC_01/95 genome structure shows a continuous distribution of genes. The sparse region of the genome, in which effectors are commonly found, is considered to be plastic and to be responsible for host adaptability (Raffaele et al. 2010), and the majority of our identified CREs were located in the sparse regions (Fig. 3).

\section{Five $P$. parasitica CREs are highly expressed in vitro and in planta.}

A RNA-seq analysis was performed after treating $P$. parasitica growing in liquid culture with citrus root extracts. This experiment was designed to scrutinize candidate effectors that are up-regulated in a very early step during $P$. parasitica-citrus interaction, mimicking a situation in which the microorganism perceives plant signals early and activates effectors to start the infection. We treated the mycelia of $P$. parasitica with root extracts from Citrus sunki (Sunki mandarin, a susceptible host) or Poncirus trifoliata (trifoliate orange, a resistant host), and we found that the majority of the 172 CREs were down-regulated in both hosts. However, five putative RxLRs were up-regulated in at least one timepoint sampled after treatment with $C$. sunki root extract (Fig. 4A) and were named as PpRxLR1, PpRxLR2, PpRxLR3, PpRxLR4, and PpRxLR5. These five upregulated CREs are plotted in the sparse region on the genome architecture of $P$. parasitica (Fig. 3). PpRxLR1, PpRxLR3, and PpRXLR4

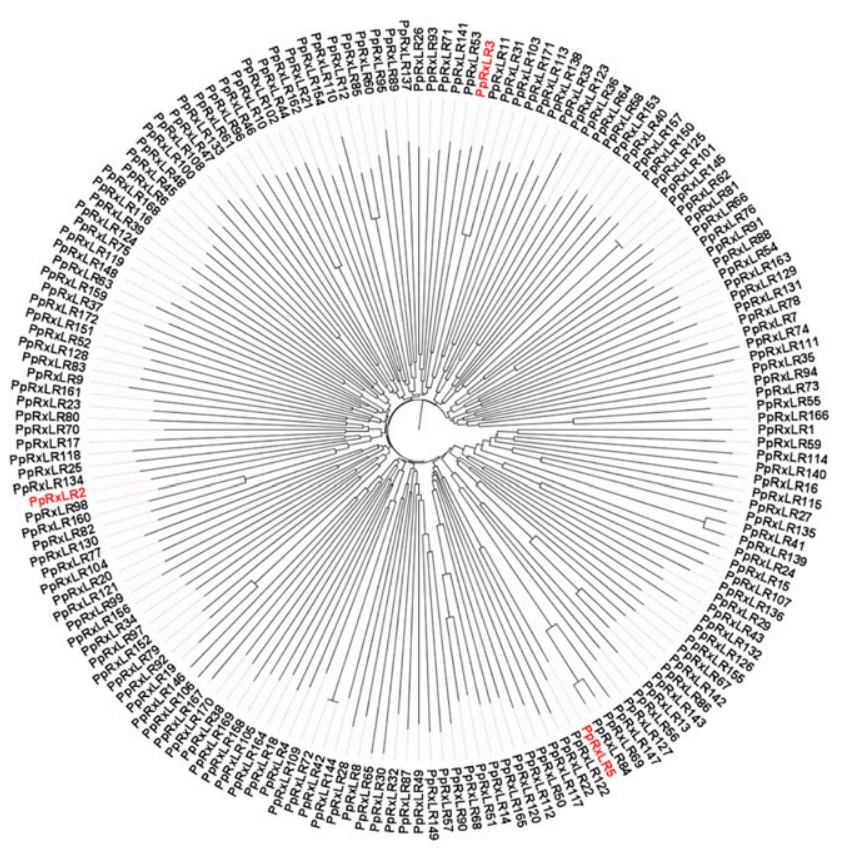

Fig. 2. Monophyletic phylogenetic tree by the neighbor-joining method showing all 172 candidate RxLR effectors of Phytophthora parasitica isolate IAC_01/95. Three RxLR candidate effectors selected for functional characterization are distributed in distinct clades. were also up-regulated after treatment with the resistant material (Fig. 4A). Subsequently, we inoculated $P$. parasitica zoospores in C. sunki and Poncirus trifoliata seedlings and analyzed gene expression of the five upregulated CREs that were identified by RNA-seq. These five CREs were strongly up-regulated during the interaction with the susceptible host, with PpRxLR1 and PpRxLR4 being highly expressed in all timepoints (Fig. 4B). During the interaction with the resistant host Poncirus trifoliata, with exception of PpRxLR5, all others were differentially upregulated in most timepoints (Fig. 4C). These five CREs were then selected for functional characterization assays in Nicotiana benthamiana plants. Currently, there is no effective protocol for agrotransformation of citrus plants.

\section{Agrobacterium-mediated transient expression assays reveal immunity suppression activity of PpRxLR2.}

To further characterize the selected CREs, we isolated and cloned their nucleotide sequences into the pCambia vector (Supplementary Material S4). PpRxLR2, $P p R x L R 3$, and $P p R x L r 5$ were successfully cloned into the plant-expressing vector and were used for transient expression in $N$. benthamiana leaves. We monitored the development of necrotic spots and performed diaminobenzidine (DAB) assays for analysis of the oxidative bursts by detecting the release of ROS. Transient expression of the genes encoding PpRxLR2, PpRxLR3 and PpRxLr5 did not cause necrotic lesions (Figs. 5A and C and 6A, C, E, and G), as the same pattern was found for leaves infiltrated with an empty vector (control). To check the ability of these genes to suppress plant defenses, we transiently expressed, via A. tumefaciens, the elicitin INF-1 of $P$. infestans. All leaves challenged with INF-1 developed necrotic spots at the agroinfiltrated site (Figs. 5B and $6 \mathrm{~B}$ and $\mathrm{D})$. On these leaves, the DAB assay for oxidative burst provided the visualization of brownish color at the borders of the necrotic areas, indicating that live cells were still releasing ROS (Figs. 5D and 6F and $\mathrm{H}$ ). Remarkably, no necrotic spot or release of reactive oxygen species were observed in leaves transiently coexpressing PpRxLR2 and INF-1 (Fig. 5B and D), indicating this putative RxLR has the ability to suppress PCD induced by INF-1. The other two putative RxLR, PpRxLR3 and PpRxLR5, were not sufficient to completely overcome the INF-1-induced PCD, although the areas of the necrotic spots were significantly smaller than INF-1 alone (Fig. 6B, D, F, and H), indicating that these two RxLR may also have the ability to suppress PCD but to a lesser extent. To prove that these phenotypes were not related to the levels of A. tumefaciens infiltration, we have infiltrated the empty vector together with the INF1 as well as with PpRxLRs plus INF1. No difference was recorded in comparison with results shown in Figures 5 and 6. Data about these further controls can be found in Supplementary Material S5.

\section{PpRxLR2, PpRxLR3, and PpRxLR5 enhanced virulence of $P$. parasitica in $N$. benthamiana leaves.}

To further elucidate the potential role of PpRxLR2, PpRxLR3, and PpRxLR5 in enhancing virulence of $P$. parasitica during infection, we challenged $N$. benthamiana leaves with $P$. parasitica zoospores $24 \mathrm{~h}$ after agroinfiltration of these putative RxLRs. The rationale of this experiment was to check if the additional transiently expressed effectors would enhance the aggressiveness of the pathogen, enabling faster disease development and producing stronger symptoms. The leaf tissues expressing PpRxLR2, PpRxLR3, and PpRxLR5 and subsequently inoculated with $P$. parasitica developed severe wilting and necrosis symptoms at $72 \mathrm{~h}$ postinoculation (hpi) (Fig. 7A). At this timepoint, no symptoms were visualized for leaves agroinfiltrated with the empty vector and posterior inoculation with zoospores. Similarly, no symptoms were developed in leaves agroinfiltrated with empty vector or the RxLR genes without inoculation with $P$. parasitica 
zoospores. At 144 hpi, all infected leaves showed symptoms, however these symptoms were more evident in the plants that also transiently expressed the PpRxLRs. In addition, we also quantified the $P$. parasitica genomic DNA at 72 hpi (Fig. 7B). We found significant differences in the inoculated and transformed samples compared with only inoculated plants at 72 hpi. PpRxLR2expressing leaves presented the highest significant amount of $P$. parasitica genomic DNA (100 times higher), followed by PpRxLR3 and PpRxLR5. Overall, these results show the ability of these RxLR effectors to enhance $P$. parasitica virulence toward $N$. benthamiana leaves. Details of the statistical analysis are shown in Supplementary Material S6.

Tridimensional structure prediction of PpRxLR2, PpRxLR3, and PpRxLR5.

To understand the molecular basis involved in different host responses elicited by PpRxLR2, PpRxLR3, and PpRxLR5, their tridimensional structures were predicted by homology modeling, using the nuclear magnetic resonance-based structure of P. capsici AVR3a4 (PcAVR3a4) as template. The three predicted structures presented an N-terminal disordered motif containing the RxLR domain at variable positions (Supplementary Material S7). PpRxLR2 structure showed this domain in the most similar position in relation to PcAVR3a4, and this predicted structure was also the most similar to the template regarding the characteristics of the $\mathrm{C}$-terminal effector domain. This domain is composed of four grouped $\alpha$-helices and contains at least seven amino-acid residues in P. infestans AVR3a (PiAVR3a) protein, which is described as crucial to modulate plant immunity (Armstrong et al. 2005). The amino-acid positions Lys80, Ile103, Tyr 147, Arg81, Lys85, Lys86 and Lys89 were not conserved in any of the analyzed PpRxLRs (Fig. 8A). However, the electrostatic potential in the surface of PpRxLR2 was very similar to that observed in the PiAVR3a predicted tridimensional structure (Fig. 8B).

\section{DISCUSSION}

The $P$. parasitica genome encodes approximately 200 potential PpRxLR effectors. This high number of secreted proteins hints at their potential role in virulence. Here, by integrating diverse genomics and transcriptomics data, we selected three potential effector proteins of $P$. parasitica. After transient expression in $N$. benthamiana leaves with INF-1-induced PCD, we could envisage the role of these CREs as plant immunity suppressors. The ability of these CREs to suppress plant defenses were then validated in $N$. benthamiana leaves expressing these PpRxLR effectors during attack by $P$. parasitica, which showed an enhanced virulence of the pathogen. $N$. benthamiana is a host of $P$. parasitica, so presumably, these PpRxLR must interact with key components of the plant defense system.

The arsenal of CREs was already reported for P. infestans, Hyaloperonospora parasitica, $P$. sojae, $P$. ramorum, $P$. capsica, and $P$. palmivora (Ali et al. 2017; Lamour et al. 2007). The large repertoire of RxLR effectors might be related to rapid evolutionary rates, to cope and adapt to either environmental or host-imposed selective pressures. Patterns of accelerated rates of gene duplication at some RxLR loci were found in $P$. sojae and $P$. ramorum genomes (Jiang et al. 2006; Tyler et al. 2006). Lamour et al. (2007) affirmed that the large repertoire of RxLR effectors in plant-pathogenic oomycetes may be correlated to a wide modulation of host processes during the interaction. Why does $P$. parasitica (genome size $95 \mathrm{Mb}$ ), with a broad host range, possess 172 candidate RxLR effectors, while $P$. infestans (genome size $240 \mathrm{Mb}$ ) and $P$. sojae (genome size $95 \mathrm{Mb}$ ), both with narrow host ranges, carry such a large repertoire (716 and 672 CREs, respectively)? One hypothetical explanation is that $P$. infestans and $P$. sojae, having a more intricate relationship with their hosts, must constantly develop new effectors to stay a step ahead in the evolution of plant $\mathrm{R}$ proteins and effector recognition. In time, this coevolutionary arms race would create

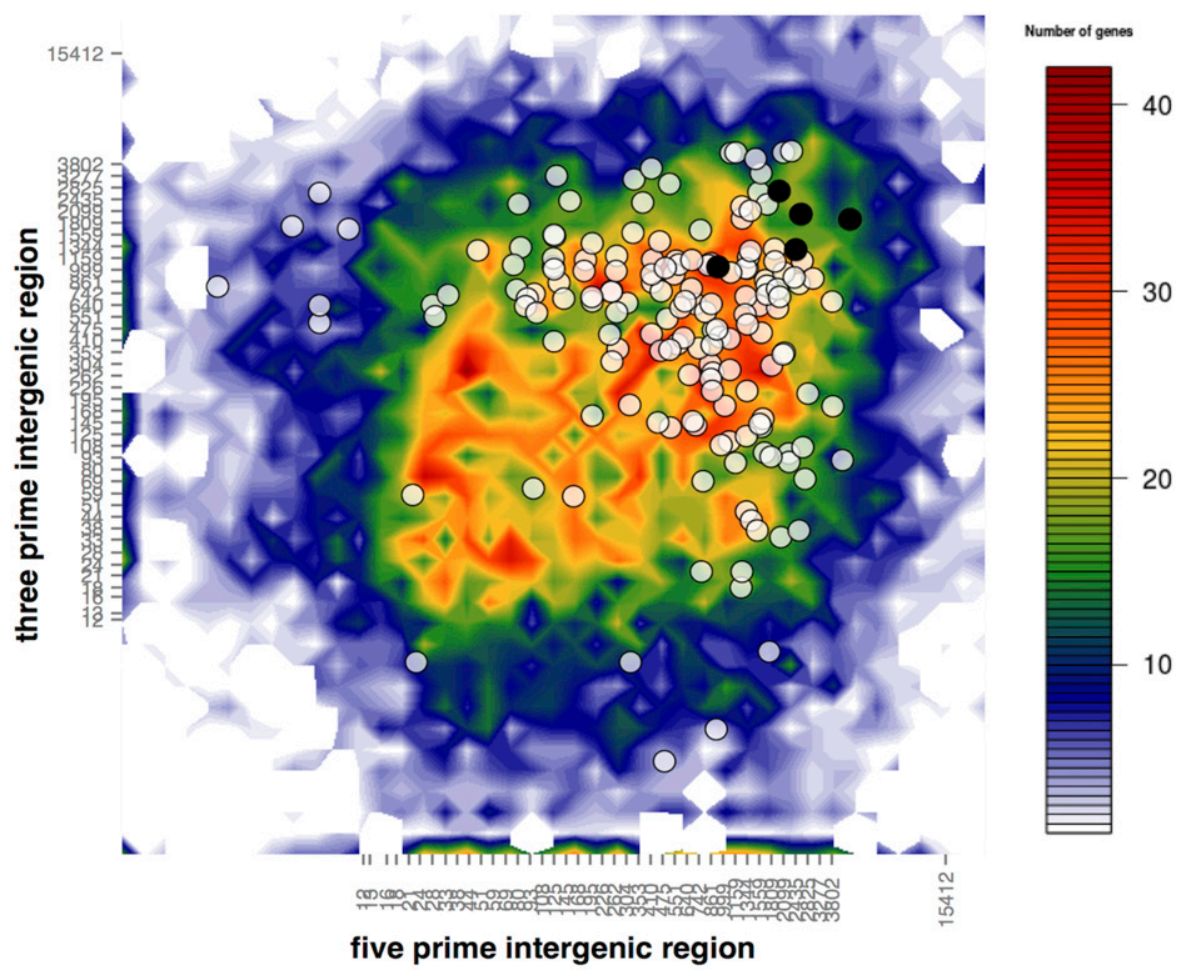

Fig. 3. Genome architecture of Phytophthora parasitica isolate IAC 01_95 with the 172 candidate RxLR effectors (CREs) plotted. Note that five upregulated genes in the transcriptomics analysis are marked in black. Most of the CREs are localized in the sparse region of the genome. The heat map shows the number of genes at the same spot on the chart. 
a more diverse repertoire of effectors. On the other hand, $P$. parasitica with a broad host range, carries a more general set of effectors, namely core effectors, which are efficient for a vast number of hosts. In addition, it would be plausible to speculate that the more general set of $P$. parasitica effectors would be a consequence of losing unutilized effector genes, as stated by the use-it-or-lose-it rule of evolution (Carroll 2006). In any case, the number of RxLR in Phytophthora spp. seems not to directly correlate with genome size, and the RxLR effectors arsenal has been shaped by host specialization and coevolutionary arms race. Ninety-three CREs were found to be present in all $P$. parasitica isolates studied herein. These core effectors probably have a more generalized and host-independent function, such as suppressing PTI. On the other hand, exclusive effectors, such as the three CREs found only in the isolate IAC 01_95 genome, may be related to more host-specific functions, such as suppressing ETI or even modulating host physiology.

The high number of CREs found in Phytophthora spp. genomes can also indicate an orchestrated secretion of these effectors. Some of them might be related to defense suppression early on the interaction, presumably in the biotrophic phase, while others may be specific to late stages of infection, in which the pathogen, being hemibiothrophic can feed on living or dead tissue. Therefore, a hierarchy among effectors is needed in which the defense suppressors would be secreted before the inducers. Wang et al. (2011), classified effectors despite the infection stage at which they are secreted, namely, immediateearly effectors (produced in the absence of haustoria) and early effectors (produced in the presence of haustoria). The authors suggest that the immediate-early effectors suppress ETI to prepare for the secretion of early effectors, whose function relies on
PTI suppression. In this study, we found five $P$. parasitica RxLR effectors expressed in vitro after treatment with root extracts. This approach mimics a hypothetical environmental scenario in which the pathogen perceives plant signals and, even before physical contact with plant cells, starts secreting effectors. These five upregulated PpRxLRs can be classified as "starter effectors," because they may play a role in manipulating plant immunity at very early stages of the interaction, before the action of immediate-early effectors. Out of 172 CREs, only five were upregulated in vitro. The strong induction of these PpRxLRs also suggests a potential role of these effectors at the early stages of infection. Accordingly, our subsequent analysis found that, with the exception of PpRxLR5, the other four PpRxLRs were strongly up-regulated during the $P$. parasitica interaction with susceptible and resistant citrus species, with three showing the ability to suppress host immunity. Altogether, we selected five of a total of 172 CREs and found that three candidate PpRXLRs contribute to pathogenicity.

These results may raise some questions about how a high rate of success can be obtained in such an under-representative library? We believe that the main answer to this question is placed on the strong upregulation of these genes even before infection, as shown by our in-vitro transcriptomics. Moreover, the five selected CREs present some interesting features that indicate they are bona fide RxLR effectors, including i) the presence of integral signal peptide amino-acid sequences, ii) the presence of the RxLR-EER motif at the N-terminal region, iii) genomic location in sparse regions of the $P$. parasitica genome, and iv) differential gene expression in planta.

PpRxLR2, PpRxLR3, and PpRxLR5 were clustered at distinct clades (Fig. 2). PpRxLR3 and PpRxLR5 were not sufficient to

A
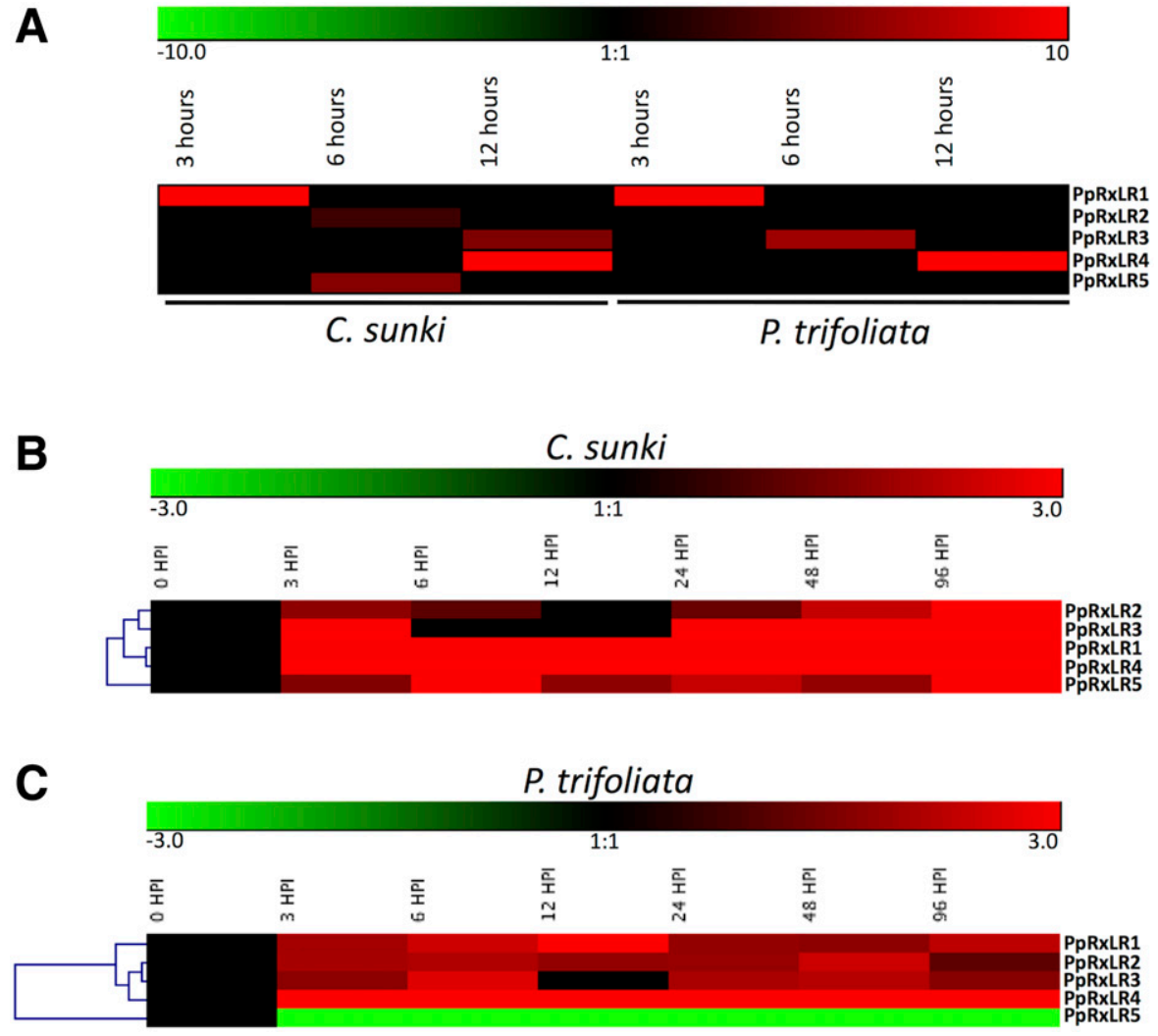

Fig. 4. A, Heat map showing levels of gene expression of five candidate RxLR effectors over time (3, 6, and 12 h) of Phytophthora parasitica isolate IAC_01/95 in RNA-seq after receiving treatment with Citrus sunki and Poncirus trifoliata root extracts. B, Heat map showing levels of gene expression of five RxLR effector candidates of $P$. parasitica over time in interaction with the susceptible host $(C$. sunki). C, Heat map showing levels of gene expression of five candidate RxLR effectors of $P$. parasitica over time in interaction with the tolerant host (Poncirus trifoliata). The data are presented as expression relative to the reference genes $S 3 A$ and $U B C$. The values of gene expression are plotted in $\log _{2}$, and different shades indicate differing levels of suppression or overexpression. 
completely suppress INF1-induced cell death, as observed in PpRxLR2 transformed leaves. Similar to our observation on PpRxLR2, P. infestans AVR3a was also shown to suppresses host cell death induced by the elicitin INF1 (Bos et al. 2009, 2010). This result corroborated with the amount of $P$. parasitica DNA quantified in $N$. benthamiana-infected leaves. This result indicates a direct correlation between the degree of defense suppression and the speed of pathogen colonization. Several RxLR effectors from other Phytophthora species have been correlated to host defense suppression, namely, AVR1b and AVR3b from $P$. sojae, AVR3a, AVRblb2, and PITG03192 from P. infestans, and ATR1 and ATR13 from Hyaloperonospora arabidopsidis (Zheng et al. 2014). The RxLR effector PITG03192 also enhanced the virulence of $P$. infestans against $N$. benthamiana (McLellan et al. 2013), which is in accordance with our result showing enhanced $P$. parasitica colonization after transient expression of PpRxLR2, PpRxLR3, and PpRxLR5. The authors indicated that it was due to the RxLR interaction with the transcription factors NAM/ATAF1,2/CUC2 (NAC)-DNA binding proteins in the host endoplasmic reticulum (McLellan et al. 2013). PpRxLR3 and PpRxLR5 showed predicted subcellular localization in the cytoplasm and a possible interaction with NAC transcription factors should be further explored. Particularly, PpRxLR2 has predicted nuclear localization and showed a high similarity with the effector PEXRD44 of $P$. infestans, which has been classified as a core effector, because its expression during infection and presence in three $P$. infestans isolates genome (Lee et al. 2014; Oh et al. 2009). Furthermore, PpRxLR2 shares similar predicted characteristics in its 3D structure with
PiAVR3a. RxLR effectors are modular proteins comprising a signal peptide, a RXLR motif at the N-terminal region, and a C-terminal effector domain. The RxLR domain acts as a translocation signal and is essential to self-deliver into the host plant cells by an unclear mechanism (Whisson et al. 2007). An external lipid phosphatidylinositol-3-phosphate (PI3P) was observed to mediate the entry of $P$. sojae Avrlb into plant and animal host cells in a RxLR-dependent way (Kale et al. 2010). However, PI3P was not required for Melampsora lini effectors AvrM and AvrL567 uptake into the plant cytoplasm, suggesting that the role of the extracellular PI3P in RXLR-mediated effector entry is not a general mechanism (Gan et al. 2010). Moreover, PIP-binding ability was observed as dependent on the characteristics of the effector domain rather than the RxLR domain of PiAVR3a (Yaeno et al. 2011). This PiAVR3a PIP-binding ability is not involved in the effector recognition by $\mathrm{R} 3 \mathrm{a}$ but is required for both suppression of INF1-induced cell death and accumulation of the E3 ubiquitin ligase CMPG1 and PiAVR3a in planta (Bos et al. 2010). CMPG1 is a key factor in INF1 elicitin-induced cell death. PiAVR3a targets and stabilizes CMPG1, blocking the initial signal transduction and regulatory signals of plant defenses (Gilroy et al. 2011). Yaeno et al. (2011) hypothesized that a possible role for this effector-PIP binding is to target CMPG1 to the endomembrane compartments and that this complex may be released in the nucleus, where PiAVR3a can stabilize CMPG1. Moreover, the PIP-binding could stabilize the effector to avoid its removal by still unknown host defense mechanisms (Yaeno et al. 2011). Interestingly, the structural prediction of PpRxLR2, PpRxLR3, and PpRxLR5 showed that only PpRxLR2

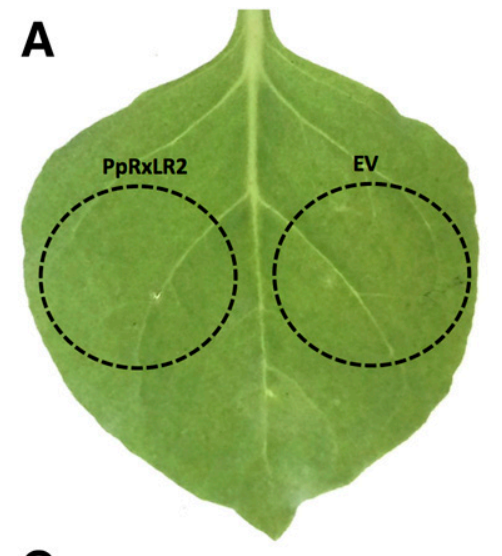

C

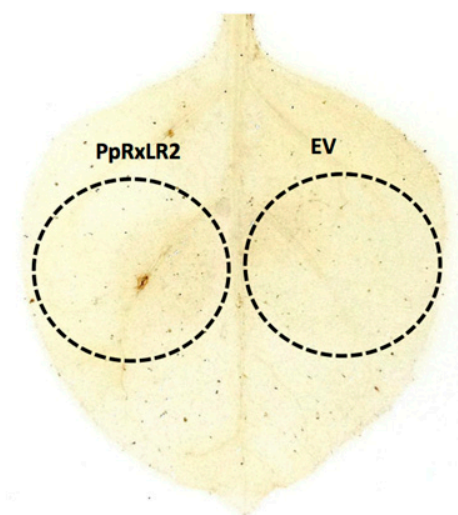

B

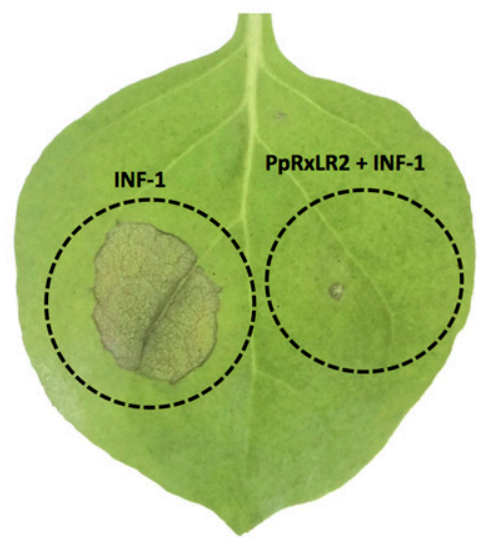

D

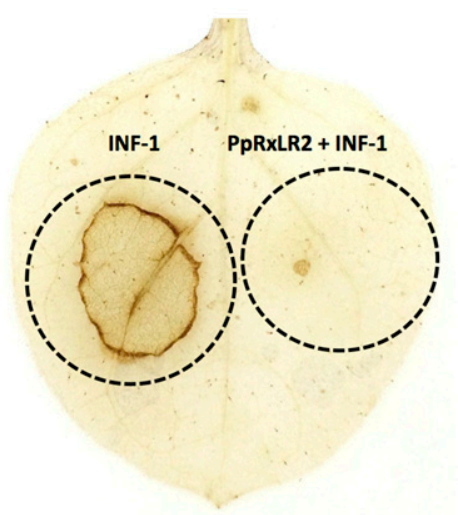

Fig. 5. Transient expression of the PpRxLR2 gene shown in adaxial views of Nicotiana benthamiana leaves, with agroinfiltation sites indicated by dotted circles. A, N. benthamiana leaves showing no necrotic lesions. EV = empty vector. $\mathbf{B}$ and $\mathbf{D}$, Hypersensitivity reaction suppression by INF-1effector PpRxLR2 after coinfiltration of INF-1 (PpRxLR2 + INF-1). The areas labeled INF-1 indicate areas of programmed cell death. C and D, Leaves treated with diaminobenzidine staining. In D, evidence of the hypersensitive response (HR) is seen by the accumulation of $\mathrm{H}_{2} \mathrm{O}_{2}$ at the transformation sites with the INF-1 gene (dark areas) and suppression of HR in the PpRxLR2 + INF-1 area. 
presents an electrostatic surface similar to PiAVR3a, whose positively charged patch is required for PIP binding (Yaeno et al. 2011) (Fig. 8). The possible interaction between PpRxLR2 and PIPs could be related to our observed responses in planta.

In summary, among the $P$. parasitica RxLR effectors, PpRxLR2 arises as the target of choice for future investigation, since it efficiently suppressed plant immunity and enhanced $P$. parasitica virulence. Further studies are necessary to uncover PpRxLR2 function in other economically important crops, such as citrus, and to decipher its mode of action for the modulation of host physiology. Finding the PpRxLR2 target host protein would reveal a plant susceptibility gene. Biotechnology-based strategies, such as gene silencing, host-induced gene silencing, and genome editing may then be applied to control $P$. parasitica diseases by targeting either PpRxLR2, its still unknown hostsusceptibility gene, or both.

\section{MATERIALS AND METHODS}

\section{$P$. parasitica growth and zoospores production.}

Phytophthora parasitica IAC 01_95 was the isolate used in all experiments in this manuscript. It was cultivated in carrotagar medium at $25^{\circ} \mathrm{C}$ in the dark. The production of sporangia was carried out according to Dalio et al. (2014). After covering $80 \%$ of the plate, the development of sporangia was induced by washing the medium with sterile water daily for a week. The release of zoospores was induced by incubating the culture at $4^{\circ} \mathrm{C}$ for an hour. A zoospore suspension was collected and its concentration was adjusted to about $10^{5}$ zoospores per milliliter for infecting citrus and $10^{6}$ zoospores per milliliter for infecting $N$. benthamiana. The number of zoospores was estimated with a Neubauer chamber.

RNA extraction, cDNA synthesis, and reverse transcription-quantitative polymerase chain reaction (RT-qPCR).

To isolate RNA total, $50 \mathrm{mg}$ of infected roots were ground to a fine powder under liquid nitrogen using a sterile mortar and pestle. Total RNA was extracted using the purification kit RNA MasterPure Planta (Epicenter Biotechnologies), according to the manufacturer's protocol, including treatment with DNase I. The RNA samples were evaluated for purity and concentration by ultraviolet spectroscopy (NanoDrop 8000; Thermo Scientific). Three biological samples were used, and all of them had RNA concentration higher than $500 \mathrm{ng} / \mu \mathrm{l}$.

The synthesis of cDNA was carried out with a mixture of $1 \mu \mathrm{g}$ of total RNA, oligo-dT primers, and M-MLV reverse transcription (Epicentre Biotechnologies). The analysis of gene expression was carried out by RT-qPCR, using the ABI PRISM 7500 (Applied Biosystems) according to the manufacturers protocol.

The RT-qPCR assays were performed with a mixture of $0.05 \mu \mathrm{g}$ of cDNA, $0.5 \mu \mathrm{M}$ of specific primers, and Absolute SYBRGreen ROX (AB Gene). The reactions were carried out with the following thermal cycler program for 40 cycles: $94^{\circ} \mathrm{C}$ for $30 \mathrm{~s}, 60^{\circ} \mathrm{C}$ for $30 \mathrm{~s}$, and $72^{\circ} \mathrm{C}$ for $30 \mathrm{~s}$. The quantification method applied was standard curve and $U B C$ (ubiquitin) and WS21 (ribosomal protein S3A) were used as reference genes.

For statistical analysis of gene expression, quantification cycle $(\mathrm{Cq})$ and primer efficiency values were calculated from raw data (Rn values) using the software Miner (Zhao and Fernald 2005) and the relative quantification $(\mathrm{Rq})$ was calculated using $2^{-\Delta \Delta \mathrm{Cq}}$ (Livak and Schmittgen 2001). The results were analyzed with the Kruskal-Wallis test (Kruskal and Wallis 1952).

\section{Plant infection and agroinfiltration.}

Seeds of $C$. sunki and Poncirus trifoliata were germinated in sterile soil. Three months after germination, the substrate was carefully removed and the seedlings were transferred to falcon tubes containing $50 \mathrm{ml}$ of distilled water. A suspension of $10^{5}$ zoospores per milliliter was added to the recipient and was sealed with parafilm to avoid evaporation. The plants were incubated in a growth chamber at $20^{\circ} \mathrm{C}$ under a photoperiod of $12 \mathrm{~h}\left(250 \mu \mathrm{mol} \mathrm{m} \mathrm{m}^{-2} \mathrm{~s}^{-1}\right)$. Six biological replicates for each treatment were used and the assay was repeated two times. The plant samples were harvested $0,3,6,12,24,48$, and $96 \mathrm{~h}$ after
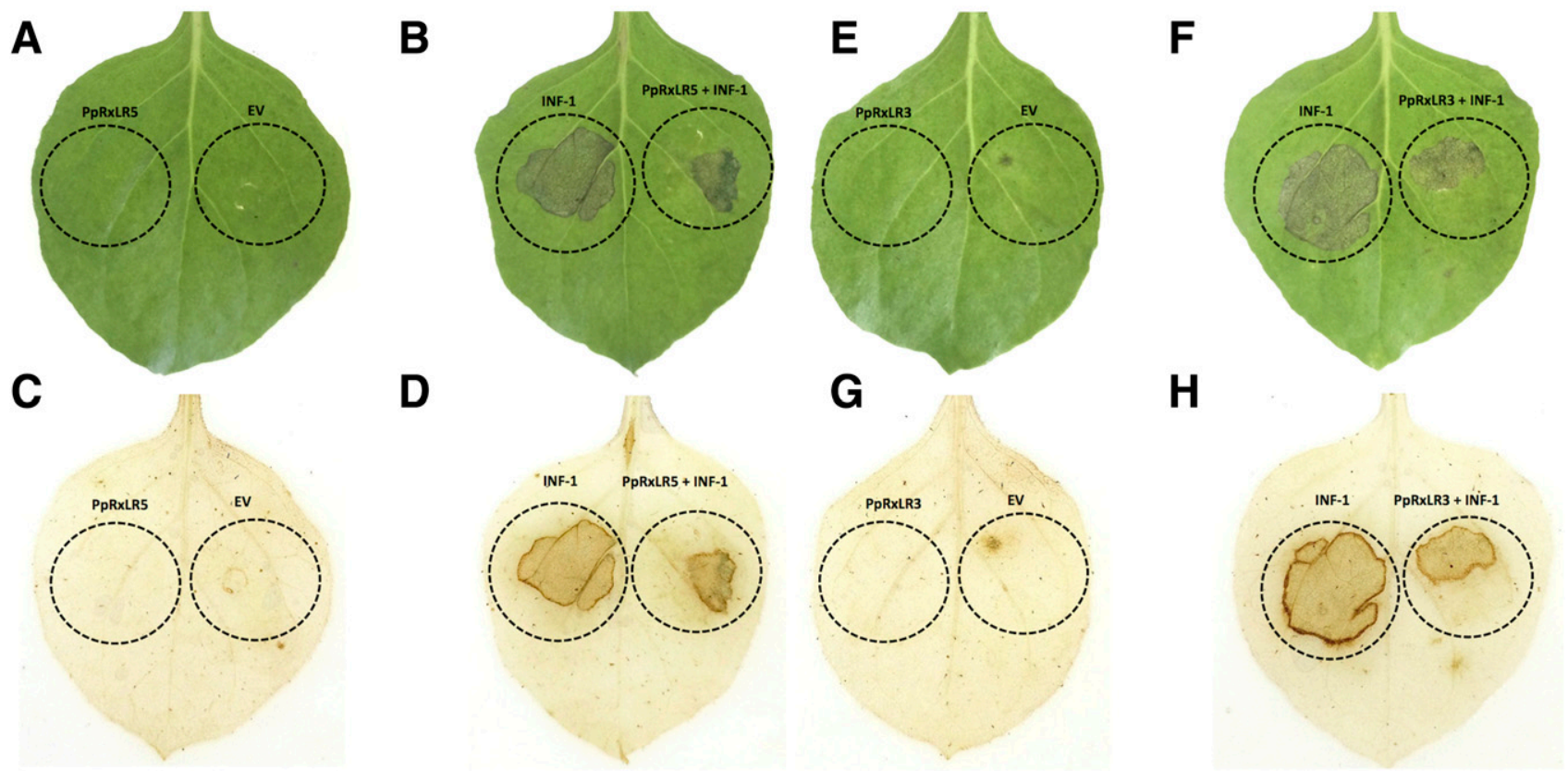

H

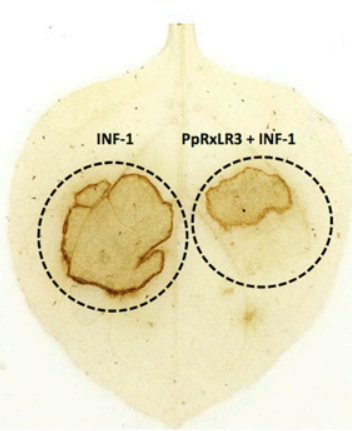

Fig. 6. Transient expression of the PpRxLR3 gene and PpRxLR5 in Nicotiana benthamiana leaves. A to D, Adaxial view of Nicotiana benthamiana leaves with agroinfiltation sites indicated by dotted circles. Partial suppression of the hypersensitivity response (HR) by the effector PpRxLR3 (B and F) and PpRxLR5 (D and $\mathrm{H}$ ) after coinfiltration with INF-1 (indicated in the areas PpRxLR3 + INF-1 and RxLR5 + INF-1). Areas of necrosis (INF-1) (B and D). E to H, leaves treated with diaminobenzidine staining. HR sites by accumulation of $\mathrm{H}_{2} \mathrm{O}_{2}$ at the transformation sites with the INF-1 gene (dark areas) ( $\mathrm{F}$ and $\mathrm{H}$ ) and partial suppression of HR in the PpRxLR3 + INF-1 and PpRxLR5 + INF-1 area (E and G). 
inoculation to analyze the expression of RxLR genes of $P$. parasitica. Agroinfiltration assays were carried out with 4- to 6-week-old Nicotiana benthamiana plants. The plants were cultivated at 22 to $25^{\circ} \mathrm{C}$ under high luminosity. Young and fully expanded leaves were used.

\section{Screening and annotation of CREs.}

Nine $P$. parasitica genomes were previously sequenced and were deposited after the Phytophthora parasitica Assembly Dev initiative, Broad Institute. To search for the RxLR candidates, we used the Galaxy tool shed (Cock et al. 2013). Basically, the algorithm searches for sequences containing signal peptide and the motif RxLR-dEER, combining strategies by Bhattacharjee et al. (2006), Win et al. (2007), and Whisson et al. (2007). After Galaxy analysis, we manually selected the positive matched sequences and performed a BlastP alignment between the sequences from the genome $P$. parasitica IAC 01_95 (query) against the sequences selected from the other eight genomes (database). The e-value 1e-07 was used as the cut-off point to ensure the minimum alignment quality.

\section{Genome architecture of $P$. parasitica.}

Evaluation of the distribution of the 172 candidate secreted effector genes in the IAC 01_95 genome was carried out by a genome architecture analysis, which was built, based on a twodimensional method of binary data, using the $\mathrm{R}$ software. This method is flexible and combines genome architecture heatmaps with scatter plots of the genomic environment and the pool of selected genes (Raffaele et al. 2010).

\section{Transcriptomics of $\boldsymbol{P}$. parasitica.}

$P$. parasitica was cultured in liquid M1 medium added with root extracts from $C$. sunki (susceptible) or Poncirus trifoliata (tolerant) (Dalio et al. 2017) at 3-, 6-, and 12-h timepoints. Root extracts were prepared by grinding $5 \mathrm{~g}$ of roots in mortar with liquid nitrogen and resuspending it on $50 \mathrm{ml}$ of phosphate

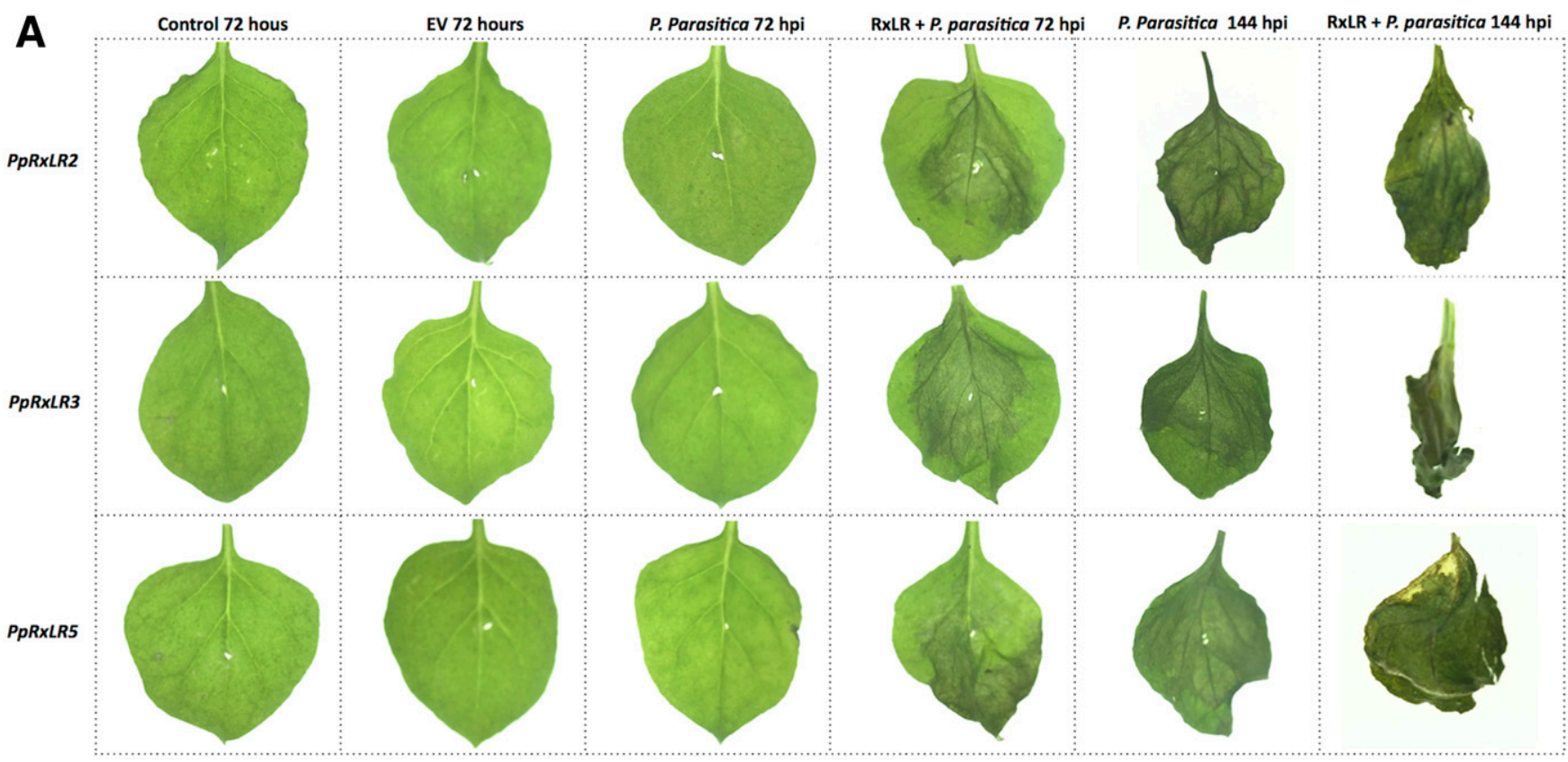

B

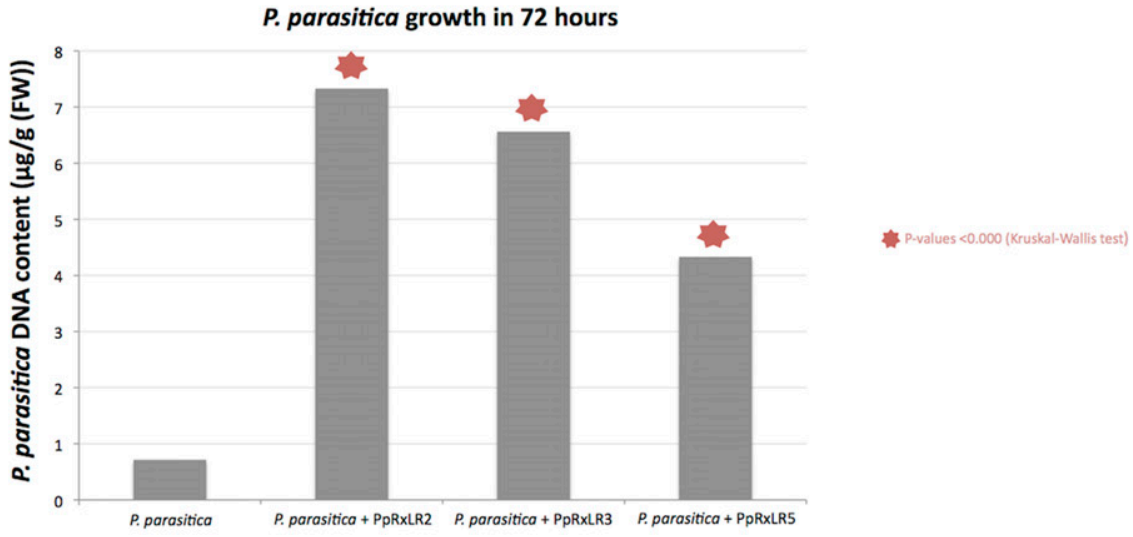

Fig. 7. A, Foliar tissues of Nicotiana benthamiana transiently transformed with the effectors PpRxLR2, PpRxLR3, and PpRxLR5 and inoculated with $10^{6}$ zoospores of Phytophthora parasitica. Foliar tissues expressing PpRxLR2, PpRxLR3, and PpRxLR5 and inoculated with $P$. parasitica developed symptoms of severe wilting and necrosis at $7272 \mathrm{~h}$ postinoculation (hpi). Controls or agroinfiltrated leaves with the RxLR genes without inoculation with $P$. parasitica zoospores and leaves infected with $P$. parasitica zoospores did not develop visible symptoms. At 144 hpi, all infected plants developed symptoms, however, these symptoms were stronger in plants that transiently expressed the PpRxLRs effectors. B, Differences in DNA detection of leaves infected with $P$. parasitica (control) and $P$. parasitica + PpRxLR2, P. parasitica + PpRxLR3, or P. parasitica + RxLR5 at 72 hpi are highly statistically significant. The amount of genomic DNA of $P$. parasitica is statistically different for control plants, with $P$ value $<0.000$, indicated by the asterisk (Kruskal-Wallis test). 
buffer, pH 7.2. After treatment, total RNA was isolated from $P$. parasitica of each treatment with the RNeasy plant mini kit (Qiagen) and was treated with DNase RNAse-free (Qiagen), according to manufacture's protocol. RNA samples were evaluated for purity and concentration, by ultraviolet spectroscopy (NanoDrop 8000), and integrity, with Bioanalyzer 1000 (Agilent). The cDNA library was sequenced by an Illumina HiSeq 2500 and the obtained reads were processed to verify

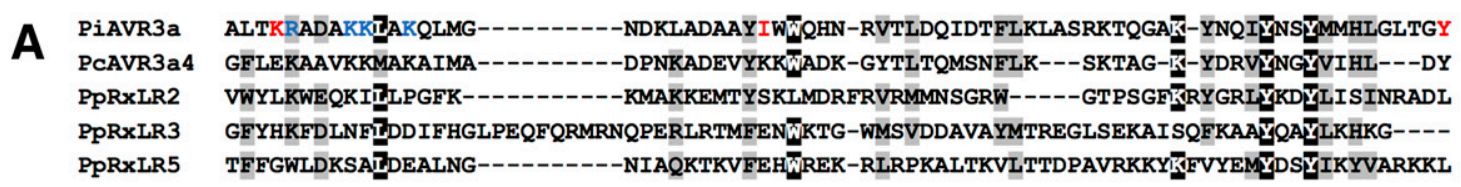

B
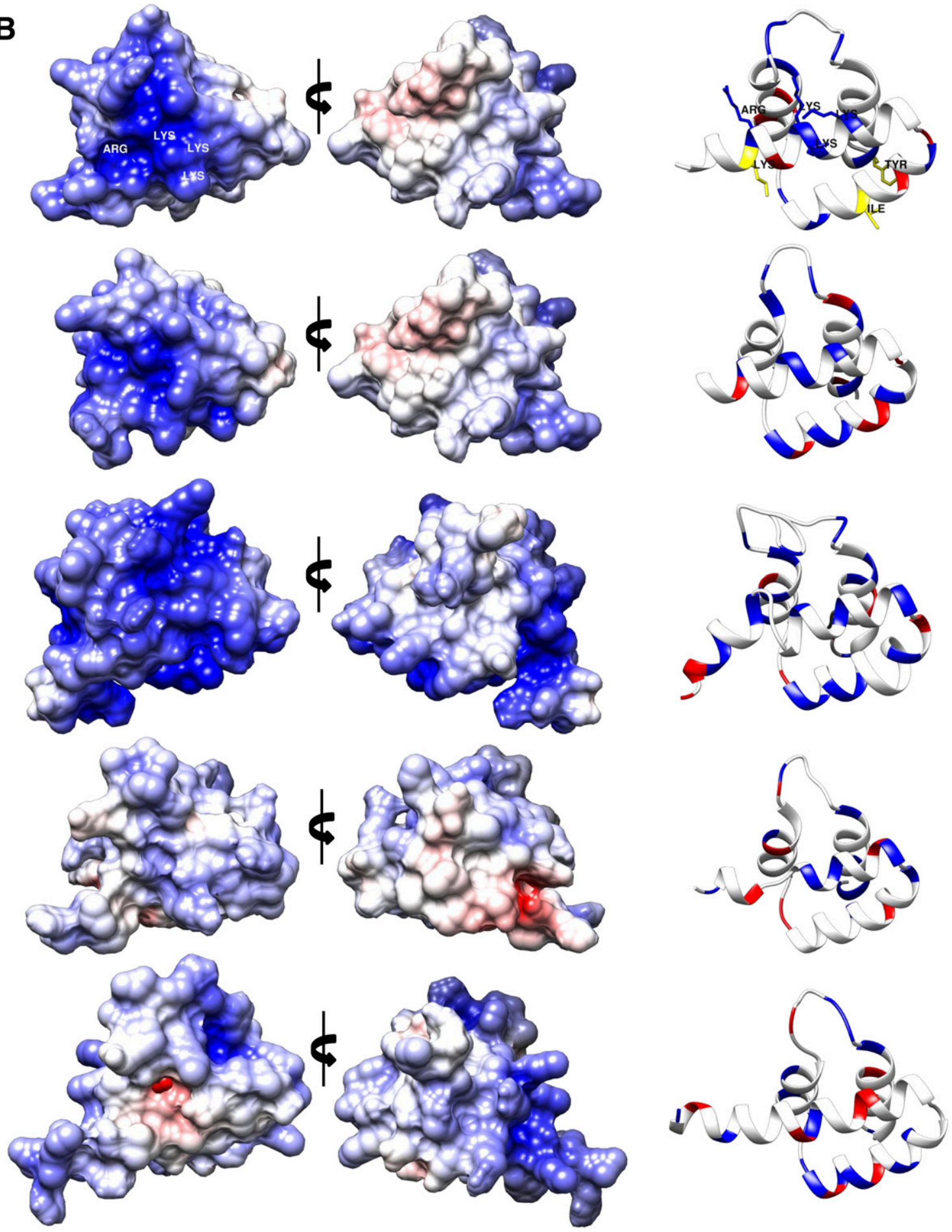

Fig. 8. Sequence and structural comparison between PiAVR3a, PcAVR3a4, PpRxLR2, PpRxLR3, and PpRxLR5 effector domains. A, Structural alignment with relevant functional positions of PiAVR3a shaded. B, Structural surfaces shaded by electrostatic potential according to the APBS calculation in the UCSF Chimera software. On the right, backbone structures illustrate positive, negative, and relevant PcAVR3a amino acids. 
their quality, in order to be used for differential expression analysis of transcripts using CLC Genomics Workbench v.6 (CLC bio).

\section{Plasmid design and agrotransformation.}

Gene sequences of PpRxLR2, PpRxLR3, and PpRxLR5 and INF1 fused to a 3xHA tag were obtained by gene synthesis and were cloned in the vector pCambia 1302. Supplementary Material S8 shows the sequences of the genes and the 3xHA (hemagglutinin) tag. The recombinant plasmids were sequenced and used for transformation of A. tumefaciens GV3101. Transformed agrobacterium were cultivated in Luria-Bertani (LB) agar plates supplemented with $50 \mu \mathrm{g}$ of kanamycin per milliliter and $50 \mu \mathrm{g}$ of rifampicin per milliliter for 2 to 3 days, at $28^{\circ} \mathrm{C}$. From a single colony on LB plate, we prepared a pre-inoculum of agrobacterium in $3 \mathrm{ml}$ of liquid LB medium with antibiotics, which was incubated in the shaker for $24 \mathrm{~h}$ at $28^{\circ} \mathrm{C}$ and $200 \mathrm{rpm}$. We used $40 \mu \mathrm{l}$ of the preinoculum to get an inoculum of 10 to $15 \mathrm{ml}$ of yeast extract broth with same antibiotics, $2 \mu \mathrm{M}$ acetosyringone, and $10 \mathrm{mM}$ morpholine ethane sulfonic acid (MES) and growth overnight till it reached the final optical density (OD) of approximately 1 . The bacteria in the medium were precipitated $(4,000 \times g$ for $10 \mathrm{~min})$, and the pellet was resuspended in MMA medium ( $20 \mathrm{~g}$ of sucrose, $5 \mathrm{~g}$ of Murashige-Skoog salts, $1.95 \mathrm{~g}$ of MES, pH adjusted to 5.6 with $\mathrm{NaOH}$, and $1 \mathrm{ml}$ of acetosyringone per liter). The final $\mathrm{OD}_{600}$ was adjusted to 0.5 to 1.0. The cells were incubated at room temperature for $3 \mathrm{~h}$. We performed infiltrations with a 1-ml syringe by pressing the needleless syringe on the underside of the leaf. Symptoms were observed 2 to 7 days after infiltration. The RxLR genes were coinfiltrated with INF-1. The assay was repeated three times.

\section{Detection of ROS.}

For detecting ROS, specifically $\mathrm{H}_{2} \mathrm{O}_{2}$ in leaves of $N$. benthamiana, we carried out the DAB assay at 5 days postinfiltration, according to Thordal-Christensen et al. (1997) and Salzer et al. (1999). The leaves were stained and were later analyzed by light-microscopy.

\section{$P$. parasitica inoculation and quantification in planta.}

To evaluate the activity of the PpRxLR2, PpRxLR3, and PpRxLR5 in the colonization and development of $P$. parasitica in $N$. benthamiana leaves, we performed agroinfiltration of these candidate effectors and further inoculated zoospores of $P$. parasitica $24 \mathrm{~h}$ after infiltration. We inoculated $1 \times 10^{6} \mathrm{zoo}-$ spores of $P$. parasitica in agroinfiltrated plants. Leaves agroinfiltrated with empty vector were used as controls. Three biological replicates were used for each treatment. The leaves were harvested $72 \mathrm{~h}$ postinoculation, after emergence of symptoms, to evaluate the amount of genomic DNA of $P$. parasitica. We ground $100 \mathrm{mg}$ of fresh mycelia in liquid nitrogen and proceeded to DNA isolation and purification using DNeasy plant mini kit (Qiagen) and further purified the DNA with Wizard Kit (Promega), according to the manufacturer's recommendation. The DNA samples were evaluated for purity and concentration by ultraviolet spectroscopy (NanoDrop 8000). We performed qPCR with a mixture of diluted DNA $\left(1: 20\right.$ in $\left.\mathrm{H}_{2} \mathrm{O}\right)$, GoTaq realtime qPCR (Promega), and $10 \mathrm{pmol}$ each of primers PN5b (5'GAACAATGCAACTTATTGGACGTT-3') and PN6 (5'-AAC CGAAGCTGCCACCCTAC-3') (Ippolito et al. 2002) in a final volume of $20 \mu \mathrm{l}$. The reactions were carried out with the following thermal cycler program: an initial denaturation at $95^{\circ} \mathrm{C}$ for $10 \mathrm{~min}$ and 40 cycles of $95^{\circ} \mathrm{C}$ for $15 \mathrm{~s}$ and $62^{\circ} \mathrm{C}$ for $60 \mathrm{~s}$. We compared the cycle threshold values with a standard curve generated from a sample with a known DNA concentration, to determine the concentration of DNA in the evaluated sample. The concentration of DNA in the standard curve ranged from
$1 \mathrm{pg}$ to $10 \mathrm{ng}$ of DNA per milliliter. The results were analyzed with the Kruskal-Wallis (Kruskal and Wallis 1952) and Wilcoxon (Bauer 1972) tests.

\section{Protein extraction and Western blot analysis.}

Leaf tissues of $N$. benthamiana were ground to a fine powder under liquid nitrogen, using a sterile mortar and pestle, and were rinsed with extraction buffer (50 mM HEPES, $150 \mathrm{mM}$ KCL, 1 mM EDTA, and $0.1 \%$ Triton X-100, with pH adjusted to 7.5 with $\mathrm{KOH}$ ) supplemented with $1 \mathrm{mM}$ dithiothreitol and protease inhibitor. The protein extracts were transferred to $15 \%$ sodium dodecyl sulfate-polyacrylamid gels, and the pattern of bands was further analyzed. The proteins were transferred to a nitrocellulose membrane. The membranes were washed in PBST (phosphate buffered saline [PBS] with $0.1 \%$ Tween 20) for $2 \mathrm{~min}$ and were blocked for $30 \mathrm{~min}$ in PBST-BSA (bovine serum albumin). Rabbit anti-HA monoclonal antibody was added to the PBST-BSA buffer and was incubated for $20 \mathrm{~min}$, using the SNAP i.d. 2.0 protein detection system method, followed by washes with PBST three times. The membranes were then incubated in PBST-BSA in addition to the chemiluminescent secondary antibody reactive to luminol.

\section{RxLRs tridimensional structure prediction.}

P. parasitica PpRxLR2, PpRxLR3, and PpRxLR5 and $P$. infestans PiAVR3a tridimensional structures were predicted by homology modeling, using Modeler software version 9.18 (Sali and Blundell 1993). For this, a total of 1,000 models each were predicted, using the atomic coordinates from the PcAVR3a4 effector from P. capsici (PDB: 2LC2) as template. The three best models predicted according to both Modeler molpdf and DOPE scores were selected and were submitted to quality assessment in the SWISS-MODEL Workspace (Arnold et al. 2006). The model presenting the highest QMEAN6 score (Benkert et al. 2008) value for each structure was selected for further analysis. Ramachandran plots predicted by the PROCHECK software (Laskowski et al. 1993) for the PpRxLR2, PpRxLR3, PpRxLR5, and PiAVR3a predicted models are presented in Supplementary Material S9. The tridimensional structures were analyzed and visualized in the UCSF Chimera software (Pettersen et al. 2004).

\section{Sequence clustering analyses.}

CRE protein sequences identified in the IAC 01_95 genome were aligned using Muscle software (Edgar 2004) and were submitted to phylogenetic inference using the neighbor-joining method (Saitou and Nei 1987) in MEGA7 software (Kumar et al. 2016). The evolutionary distances were calculated using the p-distance method (Nei and Kumar 2000). The predicted tree was visualized in Figtree software.

To identify the presence of homolog sequences to the 172 IAC 01_95 CREs in the other eight $P$. parasitica genomes, a search was conducted using the BLASTp method (Altschul et al. 1990). Homolog sequences were selected as those presenting at least $95 \%$ identity and more than $50 \%$ query coverage. The presence or absence of these sequences in each analyzed genome was used to calculate a heat map and hierarchical clustering analyses, using the heatmap. 2 function in the Gplots package (Warnes et al. 2009).

\section{ACKNOWLEDGMENTS}

We thank C. G. Litholdo and S. Pascholati for providing comments that improved the text. This work was supported by Fundação de Amparo a Pesquisa no Estado de São Paulo (Fapesp 2015/14498-6) and Conselho Nacional de Desenvolvimento Científico e Tecnológico (CNPq, 445390/ 2014-0 and 465440/2014-2). 


\section{LITERATURE CITED}

Ali, S. S., Shao, J., Lary, D. J., Strem, M. D., Meinhardt, L. W., and Bailey, B. A. 2017. Phytophthora megakarya and P. palmivora, causal agents of black pod rot, induce similar plant defense responses late during infection of susceptible cacao pods. Front. Plant Sci. 8:169.

Altschul, S. F., Gish, W., Miller, W., Myers, E. W., and Lipman, D. J. 1990. Basic local alignment search tool. J. Mol. Biol. 215:403-410.

Anderson, R. G., Deb, D., Fedkenheuer, K., and McDowell, J. M. 2015. Recent progress in RXLR effector research. Mol. Plant-Microbe Interact.28:1063-1072.

Armstrong, M. R., Whisson, S. C., Pritchard, L., Bos, J. I., Venter, E., Avrova, A. O., Rehmany, A. P., Böhme, U., Brooks, K., Cherevach, I., Hamlin, N., White, B., Fraser, A., Lord, A., Quail, M. A., Churcher, C., Hall, N., Berriman, M., Huang, S., Kamoun, S., Beynon, J. L., and Birch, P. R. 2005. An ancestral oomycete locus contains late blight avirulence gene Avr3a, encoding a protein that is recognized in the host cytoplasm. Proc. Natl. Acad. Sci. U.S.A. 102:7766-7771.

Arnold, K., Bordoli, L., Kopp, J., and Schwede, T. 2006. The SWISSMODEL workspace: A web-based environment for protein structure homology modelling. Bioinformatics 22:195-201.

Bauer, D. F. 1972. Constructing confidence sets using rank statistics. J. Am. Stat. Assoc. 67:687-690.

Benkert, P., Tosatto, S. C. E., and Schomburg, D. 2008. QMEAN: A comprehensive scoring function for model quality assessment. Proteins 71:261-277.

Bhattacharjee, S., Hiller, N. L., Liolios, K., Win, J., Kanneganti, T. D., Young, C., Kamoun, S., Haldar, K. 2006. The malarial host targeting signal is conserved in the Irish potato famine pathogen. PLoS Pathog. 2:e50.

Bos, J. I., Armstrong, M. R., Gilroy, E. M., Boevink, P. C., Hein, I., Taylor, R. M., Zhendong, T., Engelhardt, S., Vetukuri, R. R., Harrower, B., Dixelius, C., Bryan, G., Sadanandom, A., Whisson, S. C., Kamoun, S., and Birch, P. R. 2010. Phytophthora infestans effector AVR3a is essential for virulence and manipulates plant immunity by stabilizing host E3 ligase CMPG1. Proc. Natl. Acad. Sci. U.S.A. 107:9909-9914.

Bos, J. I., Chaparro-Garcia, A., Quesada-Ocampo, L. M., McSpadden Gardener, B. B., and Kamoun, S. 2009. Distinct amino acids of the Phytophthora infestans effector AVR3a condition activation of R3a hypersensitivity and suppression of cell death. Mol. Plant-Microbe Interact.22:269-281.

Carroll, S. B. 2006. The Making of the Fittest: DNA and the Ultimate Forensic Record of Evolution. W.W. Norton and Co., New York.

Cock, P. J. A., Grüning, B. A., Paszkiewicz, K., and Pritchard, L. 2013. Galaxy tools and workflows for sequence analysis with applications in molecular plant pathology. PeerJ 1:e167.

Dalio, R. J. D., Magalhães, D. M., Rodrigues, C. M., Arena, G. D., Oliveira, T. S., Souza-Neto, R. R., Picchi, S. C., Martins, P. M. M., Santos, P. J. C., Maximo, H. J., Pacheco, I. S., De Souza, A. A., and Machado, M. A. 2017. PAMPs, PRRs, effectors and R-genes associated with citruspathogen interactions. Ann. Bot. 119:749-774

Dalio, R. J. D., Fleischmann, F., Humez, M., and Osswald, W. 2014 Phosphite protects Fagus sylvatica seedlings towards Phytophthora plurivora via local toxicity, priming and facilitation of pathogen recognition. PLoS One 9:e87860.

Edgar, R. C. 2004. MUSCLE: Multiple sequence alignment with high accuracy and high throughput. Nucleic Acids Res. 32:1792-1797.

Evangelisti, E., Govetto, B., Minet-Kebdani, N., Kuhn, M. L., Attard, A., Ponchet, M., Panabières, F., and Gourgues, M. 2013. The Phytophthora parasitica RXLR effector penetration-specific effector 1 favours Arabidopsis thaliana infection by interfering with auxin physiology. New Phytol. 199:476-489.

Gan, P. H. P., Rafiqi, M., Ellis, J. G., Jones, D. A., Hardham, A. R., and Dodds, P. N. 2010. Lipid binding activities of flax rust AvrM and AvrL567 effectors. Plant Signal. Behav. 5:1272-1275.

Gilroy, E.M., Taylor, R.M., Hein, I., Boevink, P., Sadanandom, A., and Birch, P.R. 2011. CMPG1-dependent cell death follows perception of diverse pathogen elicitors at the host plasma membrane and is suppressed by Phytophthora infestans RXLR effector AVR3a. New Phytol. 190: 653-666.

Graham, J., Feichtenberger, E. 2015. Citrus phytophthora diseases: Management challenges and successes. J. Citrus Pathol. 2. Published online.

Haas, B. J., Kamoun, S., Zody, M. C., Jiang, R. H., Handsaker, R. E., Cano, L. M., Grabherr, M., Kodira, C. D., Raffaele, S., Torto-Alalibo, T., Bozkurt, T. O., Ah-Fong, A. M., Alvarado, L., Anderson, V. L., Armstrong, M. R., Avrova, A., Baxter, L., Beynon, J., Boevink, P. C., Bollmann, S. R., Bos, J. I., Bulone, V., Cai, G., Cakir, C., Carrington,
J. C., Chawner, M., Conti, L., Costanzo, S., Ewan, R., Fahlgren, N., Fischbach, M. A., Fugelstad, J., Gilroy, E. M., Gnerre, S., Green, P. J., Grenville-Briggs, L. J., Griffith, J., Grünwald, N. J., Horn, K., Horner, N. R., Hu, C. H., Huitema, E., Jeong, D. H., Jones, A. M., Jones, J. D., Jones, R. W., Karlsson, E. K., Kunjeti, S. G., Lamour, K., Liu, Z., Ma, L., Maclean, D., Chibucos, M. C., McDonald, H., McWalters, J., Meijer, H. J., Morgan, W., Morris, P. F., Munro, C. A., O’Neill, K., OspinaGiraldo, M., Pinzón, A., Pritchard, L., Ramsahoye, B., Ren, Q., Restrepo, S., Roy, S., Sadanandom, A., Savidor, A., Schornack, S., Schwartz, D. C., Schumann, U. D., Schwessinger, B., Seyer, L., Sharpe, T., Silvar, C., Song, J., Studholme, D. J., Sykes, S., Thines, M., van de Vondervoort, P. J., Phuntumart, V., Wawra, S., Weide, R., Win, J., Young, C., Zhou, S., Fry, W., Meyers, B. C., van West, P., Ristaino, J., Govers, F., Birch, P. R., Whisson, S. C., Judelson, H. S., and Nusbaum, C. 2009 Genome sequence and analysis of the Irish potato famine pathogen Phytophthora infestans. Nature 461:393-398.

Hogenhout, S. A., Van der Hoorn, R. A., Terauchi, R., and Kamoun, S. 2009. Emerging concepts in effector biology of plant-associated organisms. Mol. Plant-Microbe Interact.22:115-122.

Ippolito, A., Schena, L., and Nigro, F. 2002. Detection of Phytophthora nicotianae and P. citrophthora in citrus roots and soils by nested PCR Eur. J. Plant Pathol. 108:855-868.

Jiang, R. H. Y., Tyler, B. M., Whisson, S. C., Hardham, A. R., and Govers, F. 2006. Ancient origin of elicitin gene clusters in Phytophthora genomes. Mol. Biol. Evol. 23:338-351.

Jones, J. D. G., and Dangl, J. L. 2006. The plant immune system. Nature 444:323-329.

Kale, S. D., Gu, B., Capelluto, D. G., Dou, D., Feldman, E., Rumore, A., Arredondo, F. D., Hanlon, R., Fudal, I., Rouxel, T., Lawrence, C. B., Shan, W., and Tyler, B. M. 2010. External lipid PI3P mediates entry of eukaryotic pathogen effectors into plant and animal host cells. Cell 142: 284-295.

Kamoun, S. 2006. A catalogue of the effector secretome of plant pathogenic oomycetes. Annu. Rev. Phytopathol. 44:41-60.

Kamoun, S., Furzer, O., Jones, J. D. G., Judelson, H. S., Ali, G. S., Dalio, R. J. D., Roy, S. G., Schena, L., Zambounis, A., Panabières, F., Cahill, D., Ruocco, M., Figueiredo, A., Chen, X.-R., Hulvey, J., Stam, R. Lamour, K., Gijzen, M., Tyler, B. M., Grünwald, N. J., Mukhtar, M. S., Tomé, D. F. A., Tör, M., Van Den Ackerveken, G., McDowell, J., Daayf, F., Fry, W. E., Lindqvist-Kreuze, H., Meijer, H. J. G., Petre, B., Ristaino, J., Yoshida, K., Birch, P. R. J., and Govers, F. 2015. The top 10 oomycete pathogens in molecular plant pathology. Mol. Plant Pathol. 16:413-434.

King, S. R., McLellan, H., Boevink, P. C., Armstrong, M. R., Bukharova, T., Sukarta, O., Win, J., Kamoun, S., Birch, P. R., and Banfield, M. J. 2014 Phytophthora infestans RXLR effector PexRD2 interacts with host MAPKKK $\epsilon$ to suppress plant immune signaling. Plant Cell 26: 1345-1359.

Kruskal, H. W., and Wallis, A. W. 1952. Use of ranks in one-criterion variance analysis. J. Am. Stat. Assoc. 47:583-621.

Kumar, S., Stecher, G., and Tamura, K. 2016. MEGA7: Molecular evolutionary genetics analysis version 7.0 for bigger datasets. Mol Biol. Evol. 33:1870-1874.

Künstler, A., Bacsó, R., Gullner, G., Hafez, Y. M., and Király, L. 2016. Staying alive-Is cell death dispensable for plant disease resistance during the hypersensitive response? Physiol. Mol. Plant P. 93:75-84.

Lamour, K. H., Win, J., and Kamoun, S. 2007. Oomycete genomics: New insights and future directions. FEMS Microbiol. Lett. 274:1-8.

Laskowski, R. A., MacArthur, M. W., Moss, D. S., and Thornton, J. M. 1993. PROCHECK: A program to check the stereochemical quality of protein structures. J. Appl. Cryst. 26:283-291.

Lee, H.-A., Kim, S.-Y., Oh, S.-K., Yeom, S.-I., Kim, S.-B., Kim, M.-S. Kamoun, S., and Choi, D. 2014. Multiple recognition of RXLR effectors is associated with nonhost resistance of pepper against Phytophthora infestans. New Phytol. 203:926-938.

Livak, K. J., and Schmittgen, T. D. 2001. Analysis of relative gene expression data using real-time quantitative PCR and the $2\left(^{-\Delta \Delta C(T)}\right.$ method. Methods 25:402-408.

McLellan, H., Boevink, P. C., Armstrong, M. R., Pritchard, L., Gomez, S., Morales, J., Whisson, S. C., Beynon, J. L., and Birch, P. R. 2013. An RxLR effector from Phytophthora infestans prevents re-localisation of two plant NAC transcription factors from the endoplasmic reticulum to the nucleus. PLoS Pathog. 9:e1003670.

Nei, M., and Kumar, S. 2000. Molecular Evolution and Phylogenetics. Oxford University Press, New York.

Oh, S. K., Young, C., Lee, M., Oliva, R., Bozkurt, T. O., Cano, L. M., Win, J., Bos, J. I. B., Liu, H.-Y., van Damme, M., Morgan, W., Choi, D., Van der Vossen, E. A. G., Vleeshouwers, V. G. A. A., and Kamoun, S. 2009. In planta expression screens of Phytophthora infestans RXLR effectors 
reveal diverse phenotypes, including activation of the Solanum bulbocastanum disease resistance protein Rpi-blb2. Plant Cell 21: 2928-2947.

Panabieres, F., Ali, G. S., Allagui, M. B., Dalio, R. J. D., Gudmestad, N. C., Kuhn, M., Roy, S. G., Schena, L., and Zampounis, A. 2016. Phytophtora nicotianae diseases worldwide: New knowledge of long-recognised pathogen. Phytopathol. Mediterr. 55:20-40.

Pettersen, E. F., Goddard, T. D., Huang, C. C., Couch, G. S., Greenblatt, D. M., Meng, E. C., and Ferrin, T. E. 2004. UCSF Chimera-A visualization system for exploratory research and analysis. J. Comput. Chem. 25:1605-1612.

Raffaele, S., Win, J., Cano, L. M., and Kamoun, S. 2010. Analyses of genome architecture and gene expression reveal novel candidate virulence factors in the secretome of Phytophthora infestans. BMC Genomics 11:637.

Saitou, N., and Nei, M. 1987. The neighbor-joining method: A new method for reconstructing phylogenetic trees. Mol. Biol. Evol. 4:406-425.

Šali, A., and Blundell, T. L. 1993. Comparative protein modelling by satisfaction of spatial restraints. J. Mol. Biol. 234:779-815.

Salzer, P., Corbière, H., and Boller, T. 1999. Hydrogen peroxide accumulation in Medicago truncatula roots colonized by the arbuscular mycorrhiza-forming fungus Glomus intraradices. Planta 208:319-325.

Song, T., Kale, S. D., Arredondo, F. D., Shen, D., Su, L., Liu, L., Wu, Y., Wang, Y., Dou, D., and Tyler, B. M. 2013. Two RxLR avirulence genes in Phytophthora sojae determine soybean Rps1k-mediated disease resistance. Mol. Plant-Microbe Interact.26:711-720.

Sperschneider, J., Catanzariti, A. M., DeBoer, K., Petre, B., Gardiner, D. M., Singh, K. B., Dodds, P. N., and Taylor, J. M. 2017. LOCALIZER: Subcellular localization prediction of both plant and effector proteins in the plant cell. Sci. Rep. 7:44598

Stam, R., Jupe, J., Howden, A. J. M., Morris, J. A., Boevink, P. C., Hedley, P. E., and Huitema, E. 2013. Identification and characterisation CRN of effectors in Phytophthora capsici shows modularity and functional diversity. PLoS One 8:e59517.

Thomma, B. P., Nürnberger, T., and Joosten, M. H. 2011. Of PAMPs and effectors: The blurred PTI-ETI dichotomy. Plant Cell 23:4-15.

Thordal-Christensen, H., Zhang, Z., Wei, Y., and Collinge, D. B. 1997. Subcellular localization of $\mathrm{H} 2 \mathrm{O} 2$ in plants. $\mathrm{H}_{2} \mathrm{O}_{2}$ accumulation in papillae and hypersensitive response during the barley-Powdery mildew interaction. Plant J. 11:1187-1194.

Tyler, B. M., Tripathy, S., Zhang, X., Dehal, P., Jiang, R. H., Aerts, A., Arredondo, F. D., Baxter, L., Bensasson, D., Beynon, J. L., Chapman, J., Damasceno, C. M., Dorrance, A. E., Dou, D., Dickerman, A. W., Dubchak, I. L., Garbelotto, M., Gijzen, M., Gordon, S. G., Govers, F., Grunwald, N. J., Huang, W., Ivors, K. L., Jones, R. W., Kamoun, S., Krampis, K., Lamour, K. H., Lee, M. K., McDonald, W. H., Medina, M., Meijer, H. J., Nordberg, E. K., Maclean, D. J., Ospina-Giraldo, M. D., Morris, P. F., Phuntumart, V., Putnam, N. H., Rash, S., Rose, J. K.,
Sakihama, Y., Salamov, A. A., Savidor, A., Scheuring, C. F., Smith, B. M., Sobral, B. W., Terry, A., Torto-Alalibo, T. A., Win, J., Xu, Z., Zhang, H., Grigoriev, I. V., Rokhsar, D. S., and Boore, J. L. 2006 Phytophthora genome sequences uncover evolutionary origins and mechanisms of pathogenesis. Science 313:1261-1266.

Wang, Q., Han, C., Ferreira, A. O., Yu, X., Ye, W., Tripathy, S., Kale, S. D., Gu, B., Sheng, Y., Sui, Y., Wang, X., Zhang, Z., Cheng, B., Dong, S., Shan, W., Zheng, X., Dou, D., Tyler, B. M., and Wang, Y. 2011. Transcriptional programming and functional interactions within the Phytophthora sojae RXLR effector repertoire. Plant Cell 23:2064-2086.

Warnes, G. R., Bolker, B., Bonebakker, L., Gentleman, R., Liaw, W. H. A., Lumley, T., Maechler, M., Magnusson, A., Moeller, S., Schwartz, M., Venables. B. 2009. gplots: Various R programming tools for plotting data. The R Foundation, Boston. http://cran.r-project.org/package=gplots. Published online.

Whisson, S. C., Boevink, P. C., Moleleki, L., Avrova, A. O., Morales, J. G., Gilroy, E. M., Armstrong, M. R., Grouffaud, S., van West, P., Chapman, S., Hein, I., Toth, I. K., Pritchard, L., and Birch, P. R. J. 2007. A translocation signal for delivery of oomycete effector proteins into host plant cells. Nature 450:115-118.

Win, J., Morgan, W., Bos, J., Krasileva, K. V., Cano, L. M., ChaparroGarcia, A., Ammar, R., Staskawicz, B. J., and Kamoun, S. 2007. Adaptive evolution has targeted the C-terminal domain of the RXLR effectors of plant pathogenic oomycetes. Plant Cell 19:2349-2369.

Yaeno, T., Li, H., Chaparro-Garcia, A., Schornack, S., Koshiba, S., Watanabe, S., Kigawa, T., Kamoun, S., and Shirasu, K. 2011. Phosphatidylinositol monophosphate-binding interface in the oomycete RXLR effector AVR3a is required for its stability in host cells to modulate plant immunity. Proc. Natl. Acad. Sci. U.S.A. 108:14682-14687.

Zhang, M., Li, Q., Liu, T., Liu, L., Shen, D., Zhu, Y., Liu, P., Zhou, J. M., and Dou, D. 2015. Two cytoplasmic effectors of Phytophthora sojae regulate plant cell death via interactions with plant catalases. Plant Physiol. 167:164-175.

Zhao, S., and Fernald, R. D. 2005. Comprehensive algorithm for quantitative real-time polymerase chain reaction. J. Comput. Biol. 12: 1047-1064.

Zheng, X., McLellan, H., Fraiture, M., Liu, X., Boevink, P. C., Gilroy, E. M., Chen, Y., Kandel, K., Sessa, G., Birch, P. R., and Brunner, F. 2014. Functionally redundant RXLR effectors from Phytophthora infestans act at different steps to suppress early flg22-triggered immunity. PLoS Pathog. 10:e1004057.

\section{AUTHOR-RECOMMENDED INTERNET RESOURCES}

Broad Institute: www.broadinstitute.org

Figtree software: http://tree.bio.ed.ac.uk/software/figtree/

Miner software: http://ewindup.info/miner 